\title{
Increasing bandwidth of full-metal slot reflectarray antennas
}

\author{
Mikhail Sugak, Svyatoslav Ballandovich*, Grigory Kostikov, Lubov Liubina, \\ and Yury Antonov \\ Saint-Petersburg Electrotechnical University “LETI”, Saint-Petersburg, 197376, Russia
}

\begin{abstract}
Results of theoretical and experimental investigations of full-metal slot reflectarray antennas with increased bandwidth are presented. Bandwidth increasing is achieved by means of certain sizes choice for each reflective element so that the phase-error minimum in a required frequency range would be created . Two prototypes - single-layer and two-layer - of K-band slot reflectarray antennas have been manufactured and tested. Effectiveness of the described designing routine is proved by experimental data: achieved bandwidth for the single-layer and two-layer structure is $25 \%$ and $32 \%$ respectively.
\end{abstract}

\section{Introduction}

Reflectarray antennas are similar to parabolic aerials, except they have flat reflector consisting of distinct elements. Geometric parameters of each element are unique and chosen to create the required phase distribution. Thanks to that radiation patterns with a special form may be easily obtained [1-4]. This is a significant advantage of these antennas compared to the parabolic aerials. The most widespread are reflectarray antennas made with printed circuit board (PCB) technique. In this case they have another advantage - low weight. Recently, full-metal slot reflectarray antennas has become intensively investigated due to such features as the absence of dielectric losses, strength and ability to be manufactured with cost-effective techniques (laser cutting, for example). They have greater weight but other characteristics are very close to those of printed reflectarray antennas [5-8].

The main drawback of reflectarray antennas is a narrow band compared to parabolic aerials. As a result, one of the path for their development is increasing their bandwidth. In this regard, several ways to achieve this goal can be mentioned. Firstly, increasing of a number of layers or the use of complicated-shape elements; secondly, the use of optimization procedures and, thirdly, combination of the first two measures. Optimization procedures leading to the bandwidth increase are successfully used for printed multilayer reflectarray antennas designing [9]. They are based on phase-error minimization in a required frequency range. In the given paper this approach has been applied to full-metal slot reflectarray antennas - two prototypes have been designed and tested in K-band. One of them was a single-layer structure and the other was a two-layer structure.

* Corresponding author: sssr2123@yandex.ru 


\section{Optimization procedure}

\subsection{Unit-cell characteristics}

Generally, design process for reflectarray antennas can be divided into the following steps: calculation of the unit-cell characteristics; synthesis of the phase distribution; synthesis of the reflector. On the first step the local-periodicity approach is used, according to which the object of study is the unit cell (or the Floquet cell) that is a single element placed in an infinite periodic structure. This allows to take into account, to some degree, mutual coupling between elements. Despite the fact that the real reflector is not a periodic structure the effectiveness of this method has been proved by lots of full-wave simulations and experiments.

In the given paper two different reflective elements are considered: single-layer crossed dumbbell slots and two-layer square-loop slots. These elements are shown in Fig. 1 (a) and Fig. 1 (b) respectively. The main characteristic of the unit cell is a dependence of the
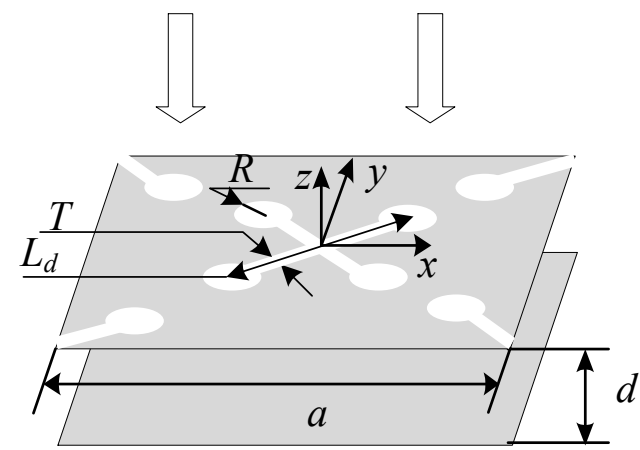

(a)
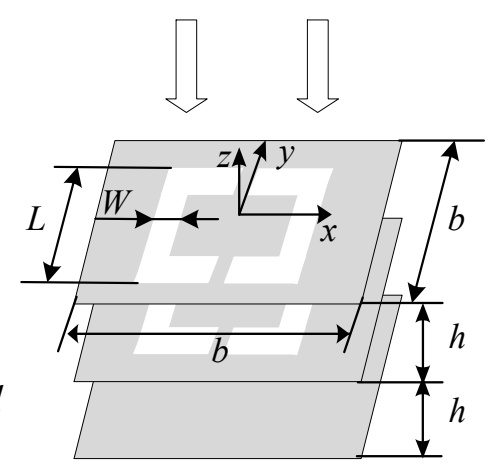

(b)

Fig. 1. Unit cell: (a) with single-layer crossed dumbbell slots, $a=8.85 \mathrm{~mm}, d=9 \mathrm{~mm}$; (b) with two-layer square-loop slots, $b=12 \mathrm{~mm}, h=3 \mathrm{~mm}, L_{d}=5.71 \mathrm{~mm}$.

reflection-coefficient phase on geometric parameters of the element. For better optimization procedure efficiency at least two parameters should be considered. In Fig. 2 phase characteristics for crossed dumbbell slots are shown in a range of $19 \ldots 23 \mathrm{GHz}$. The following general tendencies can be noted: the phase-shifting range decreases while $T$ grows and $R$ is constant; the phase-shifting range increases while $R$

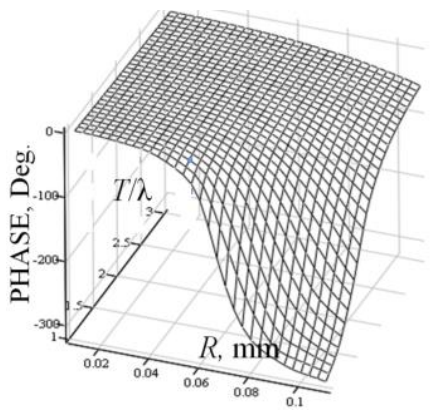

(a)

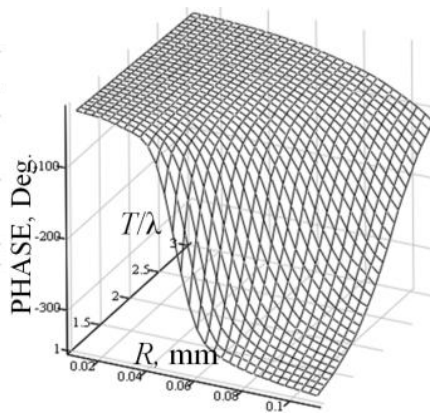

(b)

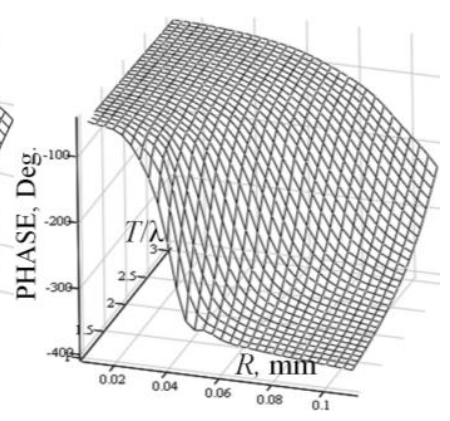

(c)

Fig. 2. Reflection-coefficient phase dependence on $R$ and $T / \lambda$ for crossed dumbbell slots at:

(a) $19 \mathrm{GHz}$; (b) $21 \mathrm{GHz}$; (c) $23 \mathrm{GHz}$. 


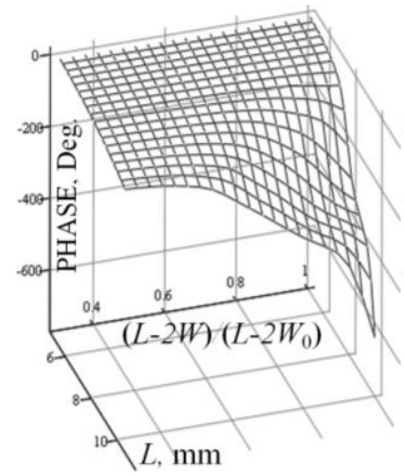

(a)

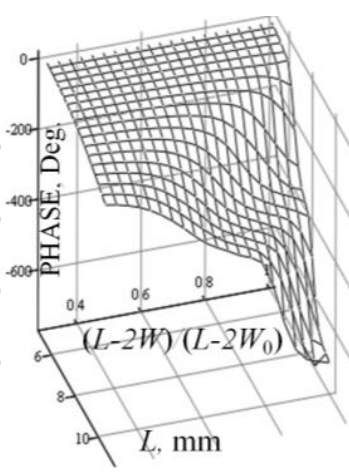

(b)

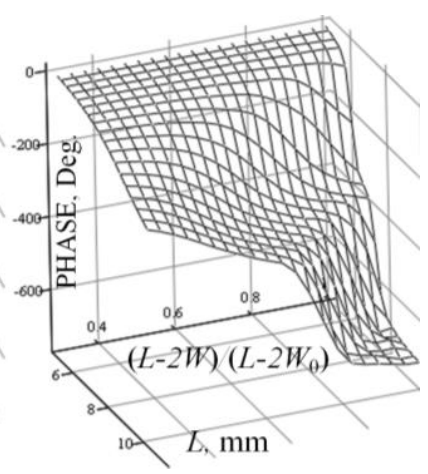

(c)

Fig. 3. Reflection-coefficient phase dependence on $L$ and the slot width for two-layer square-loop slots. The slot width is recalculated in $(L-2 W) /\left(L-2 W_{0}\right)$ ratio where $W_{0}=1 \mathrm{~mm}$. Frequencies are: (a) $14 \mathrm{GHz}$; (b) $16 \mathrm{GHz}$; (c) $18 \mathrm{GHz}$.

grows and $T$ is constant; the phase-shifting range increases while the frequency grows. Thus, the maximum phase-shifting range is about $310^{\circ}$ at $19 \mathrm{GHz}$ (Fig. 2 (a)); about $330^{\circ}$ at $21 \mathrm{GHz}$ (Fig. 2 (b)) and about $350^{\circ}$ at $23 \mathrm{GHz}$ (Fig. 2 (c)).

The same graphs for two-layer square-loop slots are shown in Fig. 3 in a range of $14 \ldots 18 \mathrm{GHz}$. In this case the phase-shifting range decreases when the slot width grows and $L$ is constant; the phase-shifting range increases when $L$ grows and the slot width is constant. It is seen that the two-layer structure has greater phase-shifting range - over $600^{\circ}$ versus about $300^{\circ}$ for the single-layer structure.

\subsection{Optimization procedure}

The single-frequency design supposes that geometry parameters of elements are chosen based on the data obtained at the central frequency. This results in gain decrease while the current frequency moves away from the central one. Generally, how fast the gain decreases depends on the geometric parameters of the antenna system and a number of layers $[1,2$, 10]. Introduction of the optimization procedures into the design process leads to significant improvement of the frequency band [9]. The main point is to provide appropriate change of the each-element phase characteristics in a given frequency range.

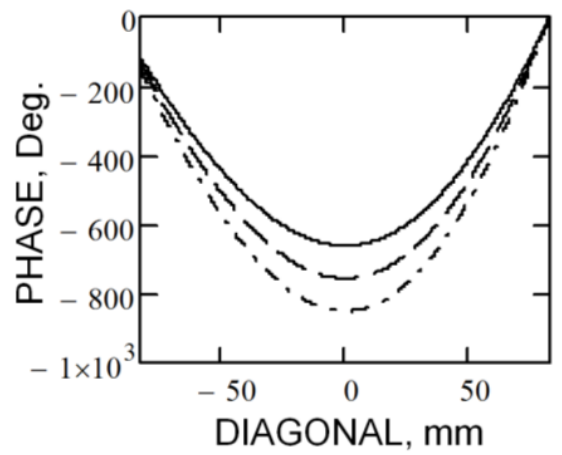

Fig. 4. The desired reflection-coefficient phase distribution along reflector with the offset feeding at $14 \mathrm{GHz}$ (solid line), $16 \mathrm{GHz}$ (dashed line) and $18 \mathrm{GHz}$ (dash-dot line).

For example, the desired reflection-coefficient phase distribution along the reflector is given in Fig. 4 for $14 \mathrm{GHz}, 16 \mathrm{GHz}$ and $18 \mathrm{GHz}$. This distribution is calculated when the 
source point is located at $0 \mathrm{~mm},-41 \mathrm{~mm}, 166 \mathrm{~mm}$ (offset angle is $14^{\circ}$, the origin of the coordinate system coincides with the reflector centre). According to the graph frequencyphase characteristics of elements should change quickly in the centre of the reflector and, in contrast, remain almost the same at the periphery. Thus, by the thorough selection of the each-element parameters the minimum difference between the required and the real reflection-coefficient phase distribution can be provided at the given frequencies. In this case the flat reflector works as the parabolic one in the given band. The process of selection of the elements parameters may be described by the following expression:

$$
\varphi_{\Sigma}=\sum_{m} \sum_{n}\left\{\sum_{i}\left|\arg \left(\operatorname{S} 11\left(x_{m}, y_{n}, f_{i}\right)\right)+\left[\varphi_{\text {inc }}\left(x_{m}, y_{n}, f_{i}\right)-\varphi_{0}\right]\right|\right\} \rightarrow \min , \ldots
$$

where $\left(x_{m}, y_{n}\right)$ coordinates of the element; $f_{i}$ - the current frequency; $\operatorname{S11}\left(x_{m}, y_{n}, f_{i}\right)$ reflection coefficient of the element; $\varphi_{\text {inc }}\left(x_{m}, y_{n}, f_{i}\right)$ - phase of a radiating-horn field at the point $\left(x_{m}, y_{n}\right) ; \varphi_{0}$ - constant subjected to optimization. Expression (1) means that the sum of phase-error absolute values should be minimized in the given frequency band.

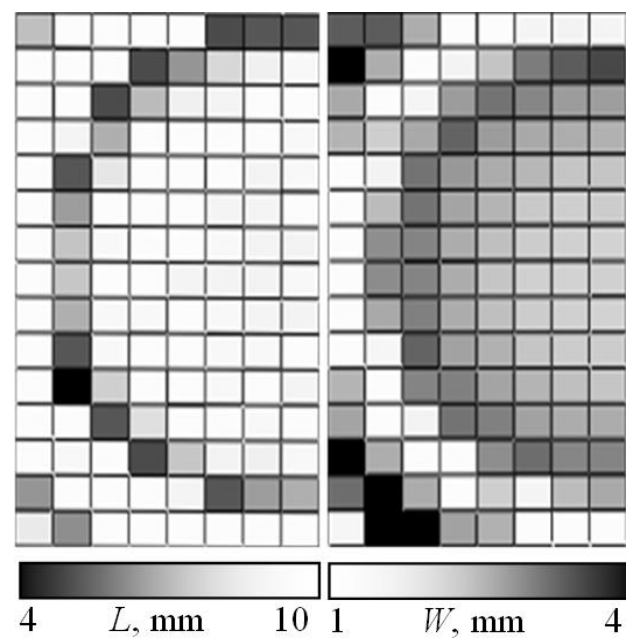

Fig. 5. A half of synthesized reflector for the two-layer offset reflectarray:

on the left - distribution of the slot length; on the right - distribution of the slot width.

Two full-metal reflectarray antennas have been optimized with the use of the described procedure. One of them is the single-layer prime-focus antenna based on dumbbell-slot elements with the following parameters: $F=143 \mathrm{~mm}$, elements are arranged in the triangular grid with the element spacing $a=8.85 \mathrm{~mm}$; the reflector has a hexagon shape with the diameter $D=186 \mathrm{~mm}$. The other one is the two-layer offset antenna based on the squareloop elements. The source-point location correspond to fig. 4, elements are arranged in the rectangular grid with the element spacing $b=12 \mathrm{~mm}$; the square reflector has a size of $168 \mathrm{~mm} \times 168 \mathrm{~mm}$. In both cases the area of the reflector is about $100 \lambda^{2}$ at the upper frequency of the operating band. It can be explained by the fact that this size with $F / D=1$ provides optimal combination of the band and the gain [10].

The synthesized geometry of the full-metal two-layer reflectarray antenna is given in Fig. 5. It is interesting to note that the slot lengths almost does not change except the vicinity of the Fresnel-zone border. In contrast, the slot width changes significantly along the reflector. If common single-frequency design process had been used behaviour of the given parameters would have been opposite. 


\section{Theoretical and experimental results}

Manufactured samples of full-metal reflectarray antennas are presented in Fig. 6. Slots are cut by laser in stainless-steel sheets $-1 \mathrm{~mm}$ thick in case of the one-layer structure and 0.5 $\mathrm{mm}$ thick in case of two-layer structure. The required distance between the ground plane and the slots is set by plastic sleeves for dumbbell slots and by layers of extruded polystyrene for square-loop slots.

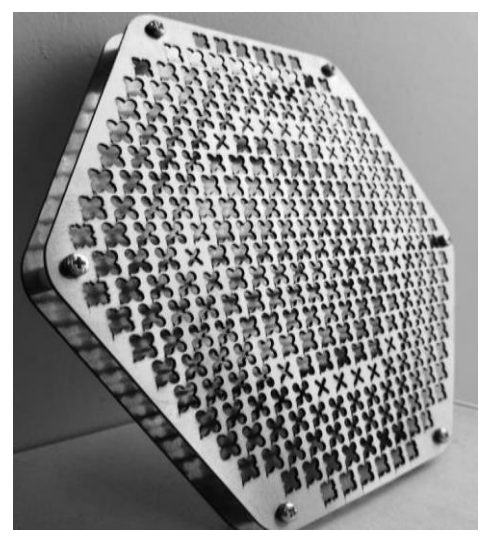

(a)

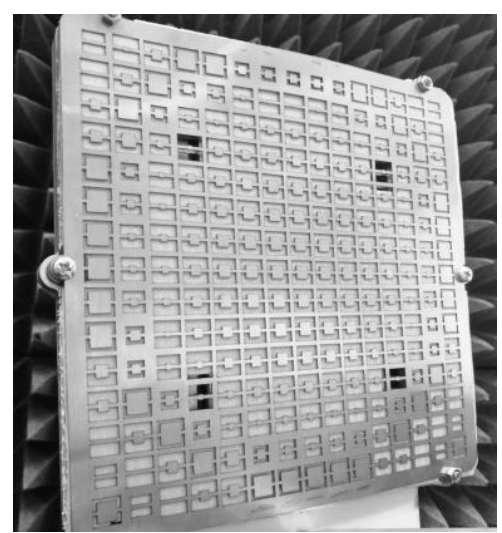

(b)

Fig. 6. Manufactured samples of full-metal reflectarray antennas: (a) based on single-layer crossed dumbbell slots; (b) based on two-layer square-loop slots.

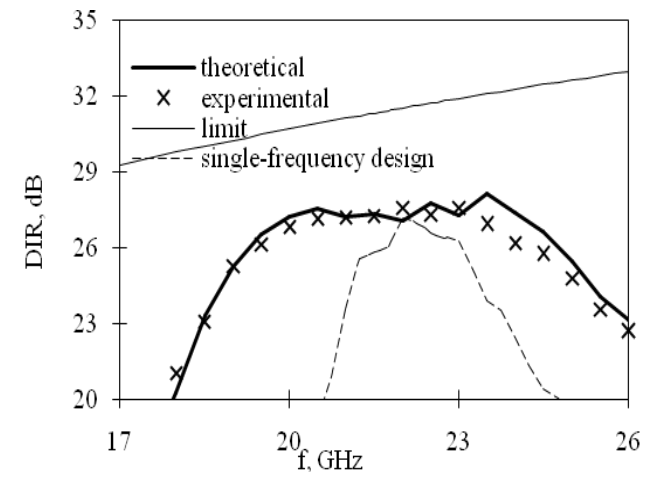

(a)

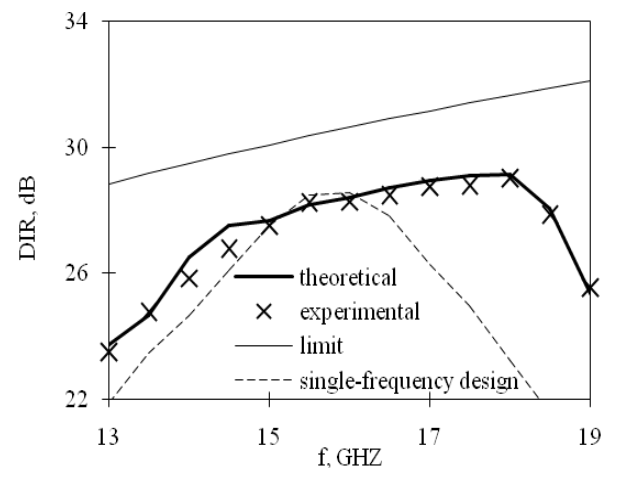

(b)

Fig. 7. Theoretical and experimental results for: (a) the single-layer antenna; (b) the two-layer antenna.

Measurements were carried out with the use of a near-field scanner. Experimental data on directivity are given in Fig. 7 (a) for the single-layer antenna and Fig. 7 (b) for the twolayer antenna. Theoretical limit corresponding to $100 \%$ aperture efficiency and directivity frequency dependence for the slot reflectarray antennas designed with the standard singlefrequency routine are given for comparison in Fig. 7 as well. Numerical data for directivity frequency dependence have been obtained from simulations in Ansys Electromagnetic Desktop programm. It is clearly seen that introduction of the optimization procedure has resulted in significant increase of the antenna-arrays' bandwidth - even for the single layer structure. Moreover, directivity increases as frequency does in the operating band, i.e. aperture efficiency remains nearly constant that is untypical for reflectarray antennas. 
Experimental aperture efficiency lies in a range of $40 \% \ldots 50 \%$ for the reflectarray antenna with dumbbell slots, for the reflectarray antenna with square-loop slots it is around $50 \%$. Relative bandwidth obtained from the experimental data is $25 \%$ for single-layer structure and $32 \%$ for the two-layer structure. These figures were calculated upon a criteria of $1 \mathrm{~dB}$ aperture-efficiency reduction. Simulations for multilayer printed reflectarray antennas based on stacked patches have been carried out as well (results are not presented). It has been found that printed antennas had better characteristics. For example, the relative bandwidth of $40 \%$ has been reached for the two-layer antenna with the $10 \lambda \times 10 \lambda$ reflector and $F / D=1$. This result is explained by better phase-frequency characteristics that printed stacked patches have.

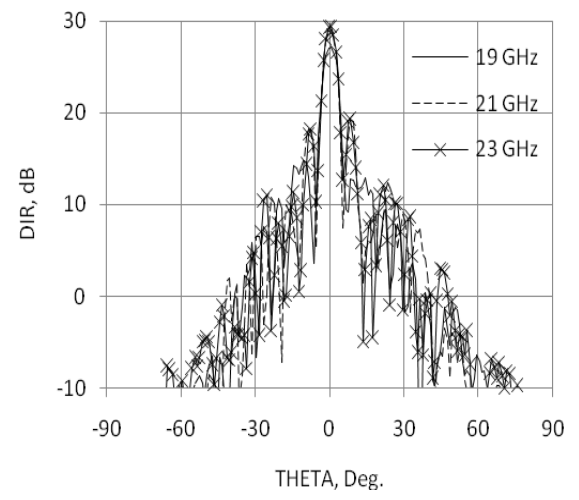

(a)

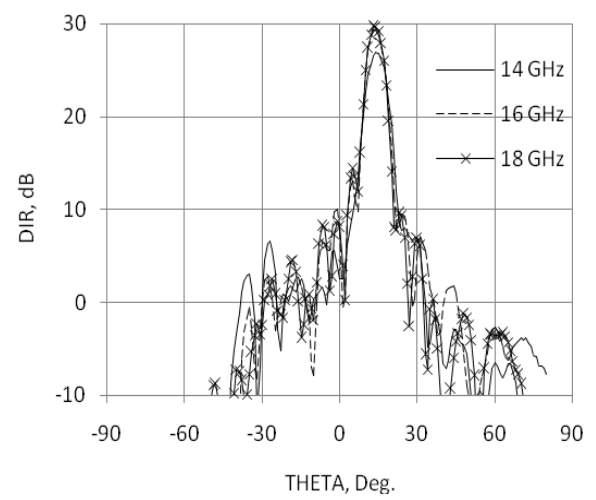

(b)

Fig. 8. Experimental radiation patterns: (a) of the single-layer antenna; (b) of the two-layer antenna.

Experimental radiation patterns in the $y z$ plane are presented in Fig. 8 (a) for the singlelayer antenna and Fig. 8 (b) for the two-layer antenna in corresponing frequency ranges. Siede-lobe level is $-10 \mathrm{~dB}$ for the first one and better than $-15.5 \mathrm{~dB}$ for the second one. From comparison of these graphs one can made a conclusion that bandwidth increases at the expense of radiation pattern quality in case of single-layer structures. At the same time, multilayer structures provide band extension with the good radiation-pattern quality.

\section{Conclusions}

Optimization procedure allowing to increase bandwidth of slot reflectarray antennas and obtain nearly constant frequency dependence of the aperture efficiency is described. This procedure is based on the careful choice of the reflective-element sizes that guarantee minimum phase error in the operating band. Effectiveness of the described optimization algorithm has been verified by means of simulations and testing two prototypes of full-metal slot reflectarray antennas. Theoretical calculations and experimental data shows that developed slot reflectarray antennas have increased bandwidth and nearly constant aperture efficiency in the operating band. Measured bandwidth of the prototypes is $25 \%$ for the single-layer structure and $32 \%$ for the two-layer structure.

\section{References}

1. J. Huang, J.A. Encinar, Reflectarray Antennas (A John Wiley \& Sons, New Jersey (2007)

2. P. Nayery, F. Yang., A.Z. Elsherbeni, Reflectarray Antennas: Theory, Designs and Applications (IEEE Press, 2018) 
3. S. V. Polenga, A. A. Erokhin, R.M. Krylov, A.V. Stankovsky, Y.A. Litinskaya, A.D. Hudonogova, I.Y. Danilov, Y.P. Salomatov, A Ka-Band Shaped-Beam Circularly Polarized Reflectarray Antenna, 2019 Radiation and Scattering of Electromagnetic Waves (RSEMW), 281-284 (June 2019)

4. S.V. Ballandovich, M.I. Sugak, G.A Kostikov, Printed reflectarray antennas with the special-form radiation pattern, Antenni, 6, 53-59 (2008)

5. W. An, S. Xuand, F. Yang, A Metal-Only Reflectarray Antenna Using Slot-Type Elements, IEEE Ant. and Wireless Propag. Letters, 13, 1553-1556 (2014)

6. S. Chen, F. Wu and J. Wang, Design of A broadband metal-only reflectarray antenna with modified slot-type elements, 2015 Asia-Pacific Microwave Conference (APMC), 1-3 (Nanjing, 2015)

7. K. Q. Henderson, N. Ghalichechian, Metal Only Spiral Slot Reflectarray Element Operating at $66 \mathrm{GHz}, 2018$ IEEE International Symposium on Antennas and Propagation \& USNC/URSI National Radio Science Meeting, 1633-1634 (Boston, MA, 2018)

8. S. V. Ballandovich, L. M. Liubina and M. I. Sugak, Investigation of slot reflectarray antennas, 2018 Moscow Workshop on Electronic and Networking Technologies (MWENT), 1-4 (Moscow, 2018)

9. M. R. Chaharmir, J. Shaker, and H. Legay, Broadband design of a single layer large reflectarray using multi cross loop elements, IEEE Trans. Antennas Propag., 57, 33633366, (2009)

10. S. V. Ballandovich, G. A. Kostikov, M. I. Sugak and Y. G. Antonov, Printed reflectarray antennas investigation based on the finite-infinite structures model, 2016 IEEE NW Russia Young Researchers in Electrical and Electronic Engineering Conference (EIConRusNW), 396-400 (St. Petersburg, 2016) 